\title{
A surgical approach to linear scleroderma using Medpor and dermal fat graft
}

\author{
Keun Tae Kim, \\ Hook Sun, \\ Eui Han Chung \\ Department of Plastic and \\ Reconstructive Surgery, Busan Paik \\ Hospital, Inje University School of \\ Medicine, Busan, Korea
}

\begin{abstract}
Linear scleroderma en coup de sabre (LScs) is a variant of localized scleroderma. This disease typically occurs in patients in their 20s or younger individuals and predominantly occurs in the forehead area. A 26-year-old man with linear scleroderma was surgically treated at our center with Medpor (porous polyethylene) and dermal fat graft for the forehead lesion. After 26 months of postoperative follow-up, the depressed lesion that appeared scarred as well as the margins improved significantly. The surgical treatment of LScs using Medpor and dermal fat graft is an effective treatment modality that can increase patient satisfaction.
\end{abstract}

Keywords: Fat / Graft / Linear / Localized scleroderma / Scleroderma

\section{INTRODUCTION}

Scleroderma is a rare disease of an unknown cause that thickens and hardens the skin due to excessive collagen deposition. Linear scleroderma en coup de sabre (LScs) is a variant of localized scleroderma that is known to occur mostly in individuals in their 20s or younger individuals. The incidence of localized scleroderma is three per one million, and $40 \%$ are of the linear subtype [1]. Localized scleroderma is more common in Caucasian women, with a female-to-male ratio of 2-4:1. Its prevalence is similar in children and adults. This disease causes depressed lesions resembling scars around the scalp and forehead. These lesions are related to the atrophy of the surrounding skin and subdermal tissues [2]. Herein, we report a case of LScs surgically treated simultaneously with Medpor (porous polyethylene) and dermal fat graft for the atrophied lesion of the forehead.

\section{CASE REPORT}

A 26-year-old man presented with scarring of the forehead and scalp. Both the patient and his family assumed the lesion to be a

Correspondence: Eui Han Chung

Department of Plastic and Reconstructive Surgery, Busan Paik Hospital, Inje

University School of Medicine, 75 Bokji-ro, Busanjin-gu, Busan 47392, Korea

E-mail: jeongyahn@gmail.com

Received June 15, 2018 / Revised July 18, 2018 / Accepted August 6, 2018 scar from a childhood trauma, but presented to our center because they thought the scar had been enlarging and worsening since the patient's teenage years. Upon observation, there was a $15-\mathrm{cm}$-long vertically depressed scar extending from the scalp toward the forehead that showed characteristic LScs findings of atrophied skin and subdermal tissues. The shape of the forehead was asymmetrical with a depression on the midline of the forehead close to the glabella. The involved skin area is depressed, hard, and hyperpigmented and may accompany hairless patches (Fig. 1A).

No hemifacial atrophy or neurological symptoms were observed, and generalized plaque morphea was noted in the left trunk. In plaque morphea, slightly raised, oval, or round areas of thickened, waxy looking skin developed slowly and spread outward. The center of the plaques was white or ivory colored (Fig. 1B). Facial computed tomography (CT) showed flattened frontal bone underlying the lesion. The size of depression identified on CT results was $35 \mathrm{~mm} \times 17 \mathrm{~mm} \times 1.9 \mathrm{~mm}$ (Fig. 2).

An incision was made on the scalp to prevent incision scars on the forehead, and dissection was performed under general anesthesia. Dissection was performed on the periosteum of frontal bone with depression. Medpor Ultra Thin sheet (oval shape $38 \mathrm{~mm} \times 50 \mathrm{~mm} \times 0.85 \mathrm{~mm}$; Stryker, Kalamazoo, MI, USA) prepared to match the width of the depression and two sheets were stacked to match the height, which was placed be- 

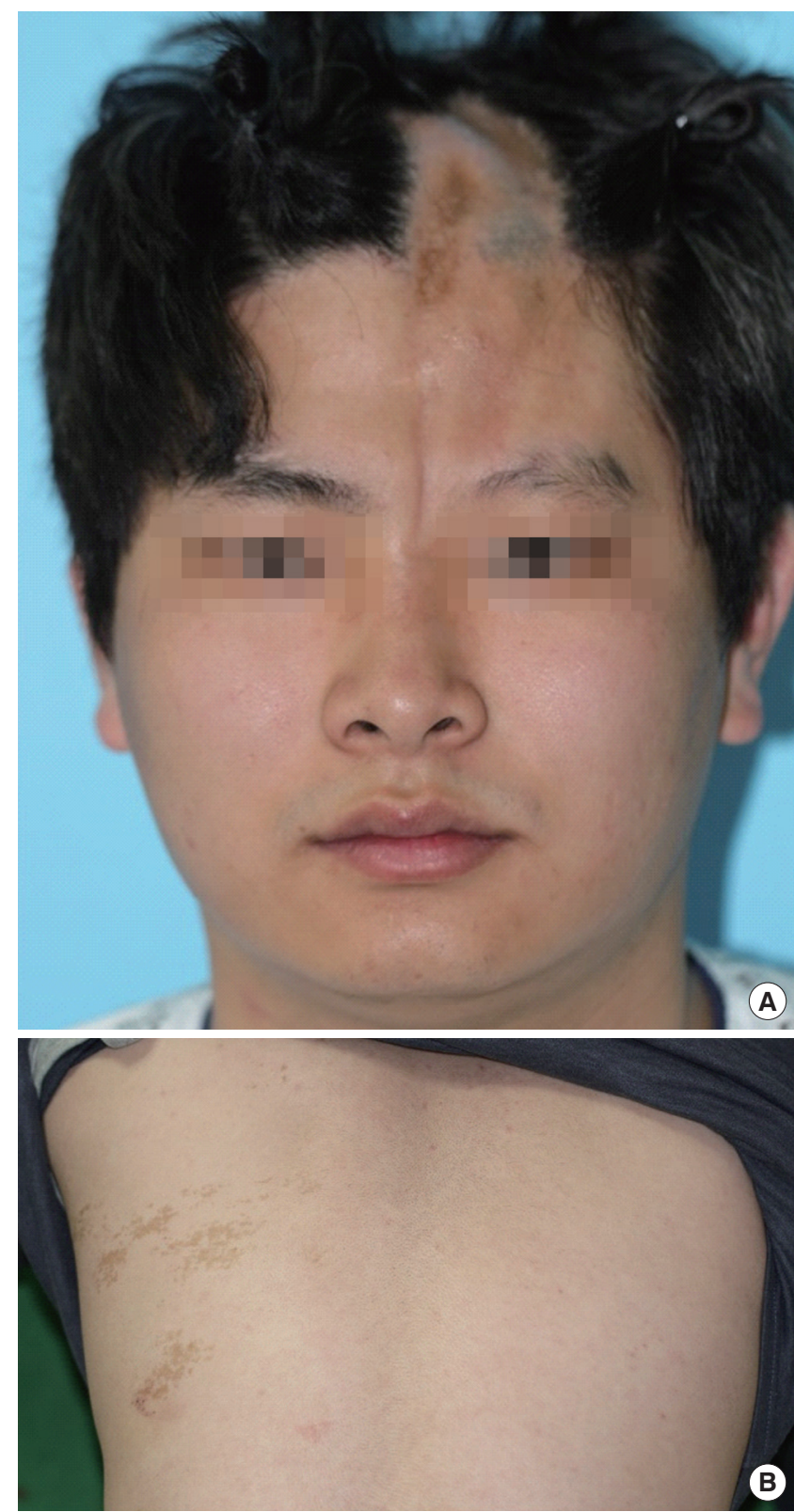

Fig. 1. Preoperative views. (A) A depressed scar on the mid-forehead region. (B) Generalized plaque morphea lesion involving the trunk.

low the periosteum and sutured to create a natural bone contour where the frontal bone defect had been. Subsequently, dermal fat graft was performed to improve the soft tissue contour. Dermal fat graft was harvested from the right inguinal area for de-epithelialization. The harvested dermal fat was placed with the dermis facing up (Fig. 3) and inserted into the pocket by pulling it through the inferior end of the lesion with a needle. The graft was then fixed to the skin superiorly and inferiorly with polypropylene sutures and removed 1 week later. Lastly, fat graft using abdominal fat was performed to improve the skin tone and create a more natural contour around the forehead defect area.

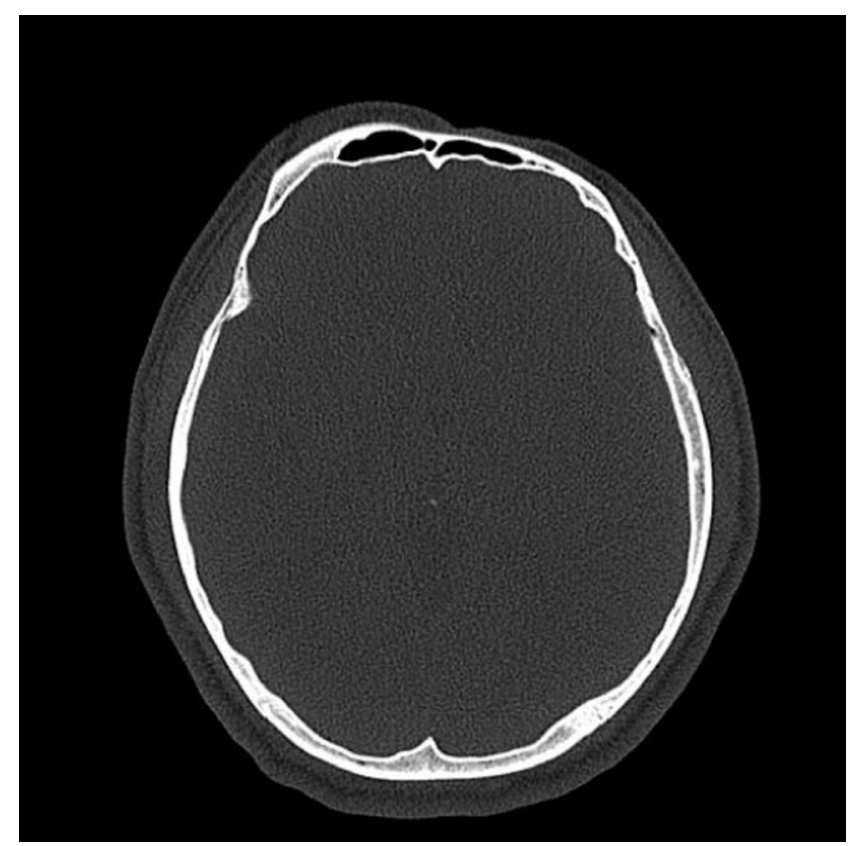

Fig. 2. Preoperative facial bone computed tomography. Soft tissue deformity with fibrotic change in the left frontal scalp and forehead.

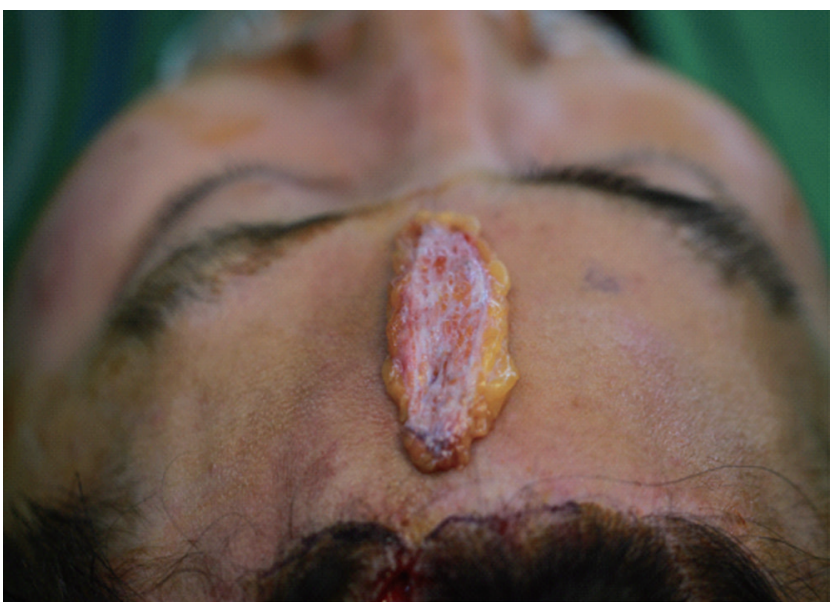

Fig. 3. Intraoperative image of the dermal fat graft.

At a follow-up visit 26 months after the surgery, the scar-like, depressed lesion and the outlines had significantly improved. The graft tissue was viable and well maintained, and patient satisfaction was quite high (Fig. 4).

\section{DISCUSSION}

LScs is characterized by atrophy and furrowing of the skin of the fronto-parietal region. The affected region is an ivory-colored depression that may be associated with hypoplasia of the underlying soft tissues and bone and result in facial hemiatrophy. As it occurs in young population and affects the forehead, linea scleroderma warrants esthetic correction [3]. 


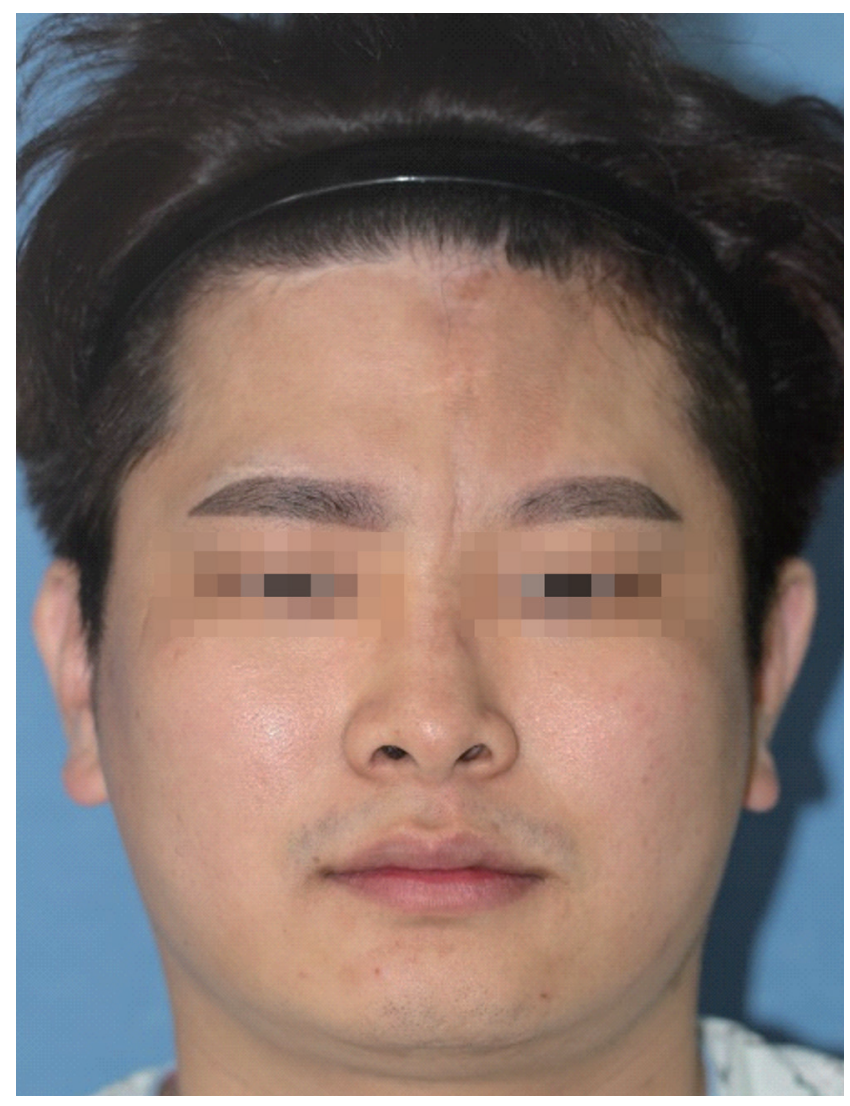

Fig. 4. At 26-month follow-up, the patient showed marked improvement.

Various treatment modalities for LScs have been introduced, such as bone graft for augmentation of the linear scleroderma at the forehead. Bone graft causes morbidity at the donor site and is limited in forming a natural appearance of the margins [4]. On the contrary, fat graft has the advantage of an autologous donor tissue, but obtaining satisfactory results is difficult in large depressed lesions. Moreover, tissue expanders provide a sufficient skin flap for closure of the defect after resection of the lesion and obtain good results. However, tissue expander takes a long time to obtain effective results, and it may be a burden to patients as it requires additional surgeries [5].

To avoid complicated procedures like those mentioned above, minimally invasive methods have been proposed as a treatment alternative. Hyaluronic acid, a soft tissue filler, is used for augmentation of the depressed scar in a patient with linear scleroderma. All filling materials have some disadvantages, including high resorption rates, repeated injections, and added cost for the fillers. For the same filling effect, autologous lipoinjection can be applied without additional cost [6]

In a recent study, a technique that uses dermal fat graft for effective treatment of linear scleroderma was introduced [5]. The method used at our hospital is similar to that method, but in the present case, Medpor was used to improve bone contour since the depression in the frontal bone was noticeable. The reason for this was Medpor implant is an excellent alternative to existing implant materials as used in our case. The implant is easy to shape, hard yet flexible, and remarkably stable, and it exhibits tissue ingrowth into its pores.

The surgical treatment provided at our center using Medpor and dermal fat graft allows simultaneous correction of the bone and soft tissue. In many cases of LScs, the lesion often extends to the scalp, which allows scar-less surgery by making the incision in an area covered by hair [7]. In addition, contour of the lesion margins can be improved more precisely through fat graft as an auxiliary procedure, while better skin tone may also be expected. Therefore, this method can be an effective treatment option for LScs with high patient satisfaction.

\section{NOTES}

\section{Conflict of interest}

No potential conflict of interest relevant to this article was reported.

\section{Ethical approval}

The study was performed in accordance with the principles of the Declaration of Helsinki. Written informed consent was obtained.

\section{Patient consent}

The patient provided written informed consent for the publication and the use of his images.

\section{ORCID}

Keun Tae Kim https://orcid.org/0000-0001-7753-739X

Hook Sun https://orcid.org/0000-0003-0104-2598

Eui Han Chung https://orcid.org/0000-0002-6581-4502

\section{REFERENCES}

1. Kimmel HL. Hemifacial atrophy. In: Rodriguez ED, Neligan PC, Losee JE, editors. Plastic surgery. 4th ed. Philadelphia: Saunders-Elsevier Health Sciences; 2017. p. 1012-4.

2. Careta MF, Romiti R. Localized scleroderma: clinical spectrum and therapeutic update. An Bras Dermatol 2015;90:62-73.

3. McKenna DB, Benton EC. A tri-linear pattern of scleroderma 'en coup de sabre' following Blaschko's lines. Clin Exp Dermatol 1999;24:467-8.

4. Hwang DY, Paik HW, Byeon JH. Correction of facial linear scleroderma 'coup de sabre' with BoneSource. J Plast Reconstr 
Aesthet Surg 2009;62:e25-8.

5. Barin EZ, Cinal H, Cakmak MA, Tan O. Treatment of linear scleroderma (en coup de sabre) with dermal fat grafting. J Cu$\tan$ Med Surg 2016;20:269-71.

6. Thareja SK, Sadhwani D, Alan Fenske N. En coup de sabre morphea treated with hyaluronic acid filler: report of a case and review of the literature. Int J Dermatol 2015;54:823-6.

7. Park SW, Wang HY. Survival of grafts in coup de sabre. Dermatol Surg 2002;28:763-6. 